\title{
"Regional tourism demand forecasting with machine learning models: Gaussian process regression vs. neural network models in a multiple-input multiple-output setting"
}

Oscar Claveria, Enric Monte, Salvador Torra 


\title{
${ }_{B}^{\mathbb{O}} \mathrm{I}|\mathrm{R}| \mathrm{E}|\mathrm{A}|$
}

Institut de Recerca en Economia Aplicada Regional i Públic

Research Institute of Applied Economics

WEBSITE: www.ub-irea.com•CONTACT: irea@ub.edu

\section{AQR}

\author{
Grup de Recerca Anàlisi Quantitativa Regional \\ Regional Quantitative Analysis Research Group \\ WEBSITE: www.ub.edu/aqr/•CONTACT: aqr@ub.edu
}

\section{Universitat de Barcelona}

Av. Diagonal, $690 \cdot 08034$ Barcelona

The Research Institute of Applied Economics (IREA) in Barcelona was founded in 2005, as a research institute in applied economics. Three consolidated research groups make up the institute: AQR, RISK and GiM, and a large number of members are involved in the Institute. IREA focuses on four priority lines of investigation: (i) the quantitative study of regional and urban economic activity and analysis of regional and local economic policies,

(ii) study of public economic activity in markets, particularly in the fields of empirical evaluation of privatization, the regulation and competition in the markets of public services using state of industrial economy, (iii) risk analysis in finance and insurance, and (iv) the development of micro and macro econometrics applied for the analysis of economic activity, particularly for quantitative evaluation of public policies.

IREA Working Papers often represent preliminary work and are circulated to encourage discussion. Citation of such a paper should account for its provisional character. For that reason, IREA Working Papers may not be reproduced or distributed without the written consent of the author. A revised version may be available directly from the author.

Any opinions expressed here are those of the author(s) and not those of IREA. Research published in this series may include views on policy, but the institute itself takes no institutional policy positions. 


\section{Abstract}

This study presents a multiple-input multiple-output (MIMO) approach for multi-step-ahead time series prediction with a Gaussian process regression (GPR) model. We assess the forecasting performance of the GPR model with respect to several neural network architectures. The MIMO setting allows modelling the cross-correlations between all regions simultaneously. We find that the radial basis function (RBF) network outperforms the GPR model, especially for long-term forecast horizons. As the memory of the models increases, the forecasting performance of the GPR improves, suggesting the convenience of designing a model selection criteria in order to estimate the optimal number of lags used for concatenation.

Keywords: Regional forecasting, tourism demand, multiple-input multiple-output (MIMO), Gaussian process regression, neural networks, machine learning

Oscar Claveria: AQR Research Group-IREA. University of Barcelona, Av. Diagonal 690, 08034 Barcelona, Spain. E-mail: oclaveria@ub.edu

Enric Monte:Department of Signal Theory and Communications, Polytechnic University of Catalunya (UPC)

Salvador Torra: Riskcenter-IREA, University of Barcelona, Av. Diagonal 690, 08034 Barcelona, Spain. 


\section{Introduction}

Machine learning (ML) methods are being increasingly used for economic and financial forecasting (Ortega and Khashanah, 2014; Von Spreckelsen et al., 2014; Stasinakis et al., 2014; Kock and Teräsvirta, 2014; Aminian et al., 2006; Medeiros et al., 2006; Chen and Leung, 2005). International tourism is becoming one of the most important economic activities worldwide, and as result there is an increasing interest in the refinement of tourism demand forecasts. A growing body of literature finds evidence in favour of a better predictive performance of machine learning models with respect to traditional forecasting methods (Adya and Collopy, 1998; Cho, 2003; Xu et al., 2016).

Statistical learning is based on the construction of computer algorithms that learn through experience. The complex nature of the data generating process of tourism demand explains the increasing use of non-linear approaches such as support vector regression (SVR) and neural network (NN) models for tourism forecasting. Akin (2015), Chen and Wang (2007), Claveria et al. (2016b) and Hong et al. (2011) all find that SVR models outperform linear models for tourism demand forecasting.

With respect to NN models, the most widely used NN feed-forward topology in tourism has been the multi-layer perceptron (MLP) network (Pattie and Snyder, 1996; Uysal and El Roubi, 1999; Law, 2000; Tsaur et al., 2002; Palmer et al., 2006; Padhi and Aggarwal, 2011; Lin et al., 2011; Teixeira and Fernandes, 2012; Claveria et al., 2015b; Molinet et al., 2015). See Athanasopoulos et al. (2011) and Song and Li (2008) for a thorough review of recent tourism demand forecasting studies.

The Radial Basis Function (RBF) network, is being increasingly used for tourism forecasting. Kon and Turner (2005) implement a RBF NN to forecast arrivals to Singapore. More recently, Cang (2014) combines RBF, MLP and SVR forecasts of UK inbound tourist arrivals in non-linear models. Subsequently, Çuhadar et al. (2014) and compare the forecasting accuracy of RBF to that of MLP networks to predict tourist demand, finding evidence in favour of RBF NNs. A complete summary on the use of NNs with forecasting purposes can be found in Zhang et al. (1998).

Originally devised for interpolation, the Gaussian Process Regression (GPR) model can be regarded as a supervised learning method based on a generalized linear regression that locally estimates forecasts by the combination of values in a kernel (Rasmussen, 1996). Gaussian process models allow to specify Bayesian priors on the data and the structure, and therefore the use of kernel analogue for machine for learning. 
Another key advantage of GPs over other statistical learning techniques is that they provide full probabilistic predictive distributions, including estimations of the uncertainty of the predictions. These features make GPR an ideal tool for forecasting purposes (Banerjee et al., 2008; Ahmed et al., 2010; Yang et al., 2013).

In spite of the fact that GPs are powerful, non-parametric tools for regression in high dimensional spaces, there is only one previous study that uses GPR for tourism forecasting (Wu et al., 2012). The authors use a sparse GPR model to predict tourism demand to Hong Kong and find that its forecasting capability outperforms those of the ARMA and SVR models. For a unifying description of sparse approximations for GPR see Quiñonero-Candela and Rasmussen (2005).

In order to fill this gap, we propose a multiple-output GPR model for multi-stepahead time series prediction. Recently, Ben Taieb et al. (2010) presented a multipleinput multiple-output (MIMO) extension of conventional local modelling approaches that allows to preserve the stochastic properties of the training series in multiple-stepahead prediction. The main aim of this study is to design a MIMO framework for multistep-ahead time series prediction with a GPR model.

To assess the forecasting performance of the presented GPR model we compare it to a RBF NN and a MLP NN used as benchmark in a MIMO setting that incorporates the cross-correlations between the inputs (international tourist arrivals to all seventeen regions of Spain) in order to generate a vector of future values (for all markets).

The study is organized as follows. The next section reviews the literature and describes the data. The third section presents the different machine learning methods applied in the study. Section four describes the experimental settings and reports the results of the multiple-step-ahead forecasting comparison. The paper concludes with some final remarks and potential lines for future research.

\section{Background and data}

As a result of the growing importance of tourism as a key driver of socio-economic progress, there is an increasing amount of literature about the contribution of tourism to economic growth (Sánchez et al., 2015; Pérez-Rodríguez et al., 2015; Chou, 2013; Schubert et al., 2011; Biagi and Pulina, 2009; Durbarry, 2004; Balaguer and CantavellaJordá, 2002). At regional level, Paci and Marrocu (2013) evaluated the impact tourism on economic growth in 179 European regions. By means of a spatial growth regression 
framework, the authors found that regional growth was positively affected by domestic and international tourism.

Most of the research on tourism at regional level in Spain focuses on three regions: Andalusia, and the Balearic and the Canary Islands. Regarding the region of Andalusia, Andrades-Caldito et al. (2013) analysed the destination image perceived by visitors over the past decade. The authors quantified the degree of convergence of each province's tourism image relative to the overall tourism image of Andalusia as a destination, and ranked the provinces according to their level of attractiveness as perceived by tourists, finding a decline in the valuation of the region's main coastal destinations in favour of those specializing in nature and culture. Similar results were obtained by SarriónGavilán et al. (2015), who used exploratory spatial data analysis techniques to analyse the impacts of tourism flows. In spite of a high degree of concentration in the littoral, the authors found a more equitable territorial distribution of tourism in coastal mature destinations and an increasing dynamism in the rural inland areas.

Bardolet and Sheldon (2008) undertook a comparative analysis between the Balearic Islands and the Hawaiian Islands, evaluating their growth paths since the 1950s, and identifying their strengths and weaknesses as archipelago destinations. While Capó et al. (2007) showed that that the continuous orientation towards tourism activities had translated in significant growth in the level of income in both the Balearic and the Canary Islands, Vera and Ivars (2009) found evidence that the impact of low-cost carriers in Spain had reinforced the specialization of tourism in real estate along the Spanish Mediterranean coast.

Aguiló et al. (2005) identified the sensitivity of the tourist market in the Balearic Islands to price changes in travel-related services. Garín-Muñoz and Montero-Marín (2007) used a dynamic model with panel data to identify and measure the impact of the main determinants of the inbound international tourism flows to the Balearic Islands from the most important source markets. The authors found that consumer loyalty to the destination was an important determinant of tourism demand. The results suggested that the demand was heavily dependent on the evolution of economic activity in each of the origin countries and on the relative cost of living of tourists in the destination.

By means of regression analysis, Rosselló et al. (2004) provided evidence of the influence of economic variables on the seasonal distribution of tourist arrivals to the Balearic Islands. The temporal concentration of tourism demand was also analysed by Duro and Farré (2015), who found significant differences across the Spanish provinces 
for the period 1999-2012. On one end, the Balearic and the Catalonian coastal provinces (with the exception of Barcelona) presented the strongest seasonal pattern, while on the other end, the Canary Islands and Madrid were the regions that showed the lowest level of seasonality. In this sense, Priego et al. (2015) used a gravity model to show that climate was an important factor in determining domestic tourism flows in Spain at regional level.

In the Canary Islands, Santana-Jiménez and Hernández (2011) used panel data analysis to evaluate the influence of the tourist's perception of overcrowding in five islands of the archipelago, obtaining an indicator of each island's maximum capacity. Focusing on the island of Tenerife, Ledesma-Rodríguez et al. (2001) estimated short-run and long-run elasticities for tourists visiting the island, obtaining significant elasticities for income, exchange rate, cost of the trip, and infrastructure. See Aguiló (2010) for an overview of tourism economics research in Spain from 2000 to 2010.

Despite the fact that most forecasting studies are conducted at the national level, several regional studies have been published in recent years. Lehmann and Wohlrabe (2015) reviewed some of the main issues related to regional forecasting. Guizzardi and Stacchini (2015) made use of business sentiment indicators form tendency surveys for real-time forecasting of hotel arrivals at a regional level, improving the forecasting accuracy of structural time series models. Rickman et al. (2009) applied a A Bayesian vector autoregression (BVAR) approach to assess whether prior information on spatial and economic linkages improved forecast accuracy of employment for the metropolitan areas of the state of Oklahoma and their proximate metropolitan areas.

Studies on tourism demand forecasting at regional level in Spain are mostly concentrated in the Balearic and the Canary Islands. Regarding the Canary Islands, GilAlana (2010) analysed the degree of persistence of monthly arrivals using different time-series approaches. Gil-Alana et al. (2008) applied seasonal unit roots and seasonally fractionally integrated models to forecast tourist arrivals to the Canary Islands, and found that simple deterministic models with seasonal dummy variables and $\mathrm{AR}(1)$ disturbances produced better results over short horizons.

For the Balearic Islands, Rosselló-Nadal (2001) predicted turning points in international visitor arrivals from the two major source markets using the leading indicator approach. Álvarez-Díaz and Rosselló-Nadal (2010) incorporated meteorological variables to forecast British tourist arrivals to the Balearic Islands. More recently, Saenz-de-Miera and Rosselló (2014) modelled tourism impacts on air pollution in Mallorca. The authors showed that the inclusion of daily stock of tourists 
improved the standard specification of urban air pollution models. By means of a Generalized Additive Model they found that a 1\% increase in tourists could be related to up to a $0.45 \%$ increase in $P M_{10}$ concentration levels.

The complex data generating process of tourism demand explains the increasing use of new non-linear approaches for tourism modelling. As a result, ML methods are experiencing a growing use in tourism forecasting (Peng et al., 2014). SVR and NN models are the most commonly used ML techniques for tourism demand prediction. Regarding SVR models, Chen and Wang (2007) predicted tourist arrivals to China with SVR, back propagation NN and ARIMA models, obtaining the best forecasting results with SVRs. Hong et al. (2011) also obtained more accurate forecasts with SVRs than with ARIMA models for Barbados. Akin (2015) compared the forecasting results of SVR to that of SARIMA and NN models to predict international tourist arrivals to Turkey, obtaining the best predictions with SVR models when the slope feature is more prominent.

There is also wide evidence in favour of NN models when compared to traditional time series models for tourism demand forecasting (Cho, 2003; Law, 2000). The first study that implemented ML techniques for tourism demand forecasting in Spain was that of Palmer et al. (2006). The authors designed a MLP NN to forecast tourism expenditure in the Balearic Islands, finding that the proposed network architecture provided more accurate forecasts when data had been detrended and deseasonalized. This result coincides with that of Claveria et al. (2017) for Catalonia, who analysed the effects of data pre-processing on the forecasting performance of $\mathrm{NN}$ models and found that the predictive accuracy of the models improved with seasonal adjusted data.

Medeiros et al. (2008) developed an alternative approach to analyse the demand for international tourism in the Balearic Islands. By using a NN model that incorporated time-varying conditional volatility and daily air passenger arrivals to Palma de Mallorca, Ibiza and Mahon as a proxy for international tourism demand for the Balearic Islands, the authors found that time-varying variances provided useful information regarding the risks associated with variations in international tourist arrivals.

Whilst NN models have been widely used in economic modelling and forecasting, other ML techniques such as GPR have been barely applied for forecasting purposes. From a wide range of ML methods, Ahmed et al. (2010) found that an MLP NN and the GPR showed the best forecasting performance on the monthly M3 time series competition data. In a similar exercise, Andrawis et al. (2011) found evidence in favour 
of a simple average combination of NN, GPR and linear models for the NN5 competition. Chapados and Bengio (2007) obtained accurate predictions of commodity spread prices by means of GPR.

GPR models can be regarded as supervised learning methods based on a generalized linear regression that locally estimates forecasts by the combination of values in a kernel (Rasmussen, 1996). The works of Smola and Barlett (2001), MacKay (2003), and Rasmussen and Williams (2006) have been key in the development of GPR models. By expressing the model in a Bayesian framework, the authors extended GPR applications beyond spatial interpolation to regression problems. Additional refinements have been proposed by Belhouari and Bermak (2004) and Girard et al. (2003) who respectively proposed using a non-stationary covariance function and the knowledge of the variance on inputs in order to improve the forecasting performance of the GPR model. However, up until now applications of GPR have been mostly restricted to a single-input singleoutput framework.

In this study, we attempt to cover this deficit by applying an extension of the GPR model for MIMO modelling, and assessing its forecasting performance at the regional level. We make use of international tourist arrivals to all seventeen regions of Spain. The MIMO GPR allows modelling the connections in tourism demand to all regions and generate a vectorial forecast. This strategy is cost-effective in computational terms, and seems particularly indicated for regional forecasting.

With this aim we use monthly data on international tourism demand used in this study are collected from the Spanish Statistical Office (National Statistics Institute INE - $\underline{\text { www.ine.es }})$. Our data set for the empirical experiment covers 183 monthly observations of of tourist arrivals at a regional level from 1999:01 to 2014:03. In spite of the fact that the forecasting performance of NNs improves when using deseasonalized data (Nelson et al., 1999), we use the raw data in order to analyse the forecasting accuracy of the models without using any pre-processing. In Figure 1 we present the mean annual growth rates of the different regions. The regions marked in grey experience a rate of growth above the average (3.7\%). These regions are located in coastal areas of the Mediterranean, showing the asymmetric concentration of tourism is Spain (Sarrión-Gavilán et al., 2015). 


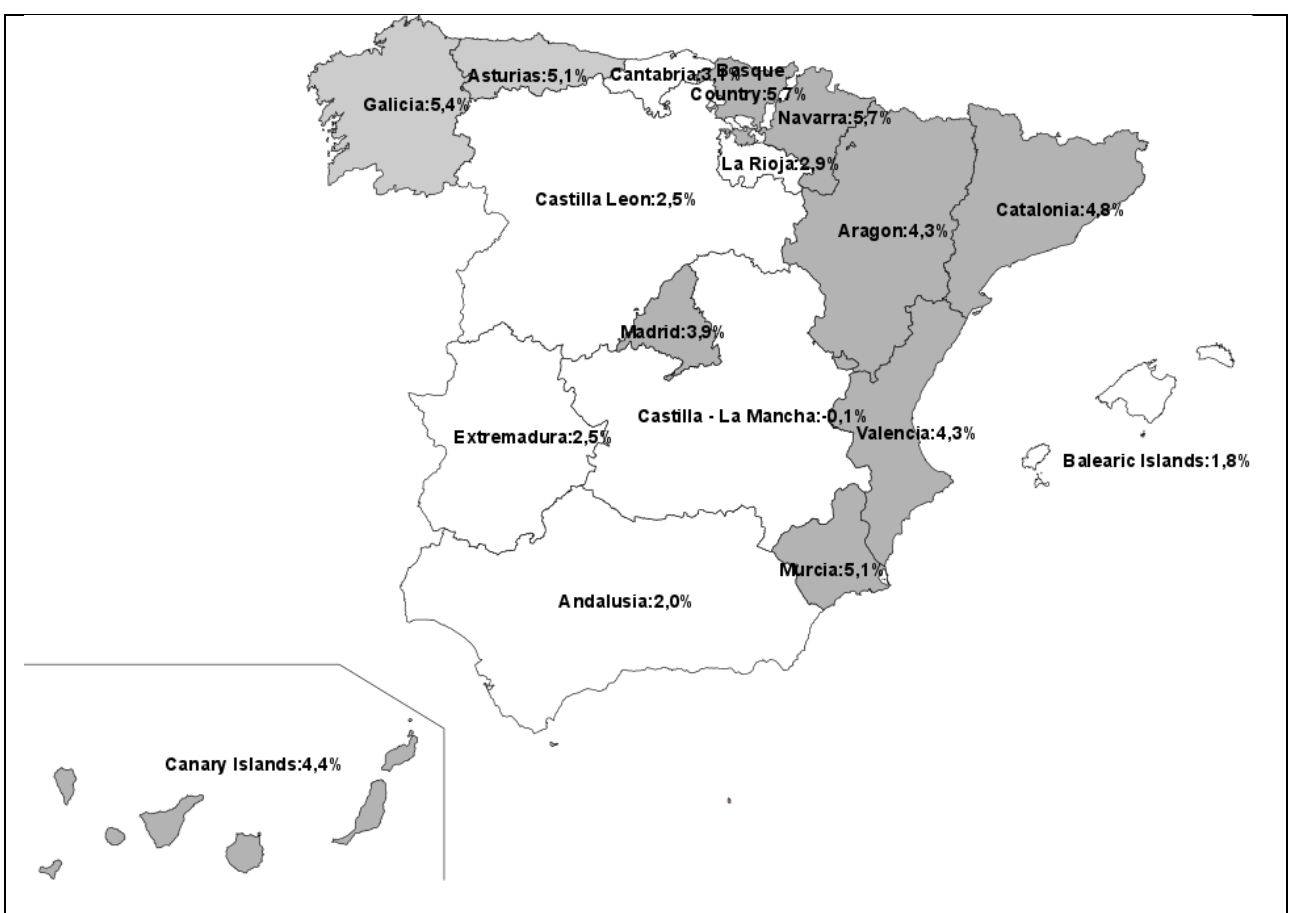

Fig 1. Mean annual growth rate of tourist arrivals to Spain by region (1999-2013)

\section{Machine learning methods}

\subsection{Gaussian Process Regression (GPR)}

GPR was first developed by Matheron (1973) based on the geostatistic works of Krige (1951). The works of MacKay (2003) and Rasmussen and Williams (2006) have been crucial in the development of GPR, which can be conceived as a method of interpolation for which the interpolated values are modelled by a GP governed by prior covariances.

By expressing the model in a Bayesian framework, different statistical methods can be implemented in GP models. Therefore GPR applications can be extended beyond spatial interpolation to regression problems. GPR is used to estimate the weights of observed values form temporal lags and spatial points using the known covariance structures. Detailed information about GPR can be found in Rasmussen and Williams (2006).

The GPR model assumes that the inputs $x_{i}$ have a joint multivariate Gaussian distribution characterized by an analytical model of the structure of the covariance matrix (Rasmussen, 1996). The key point of the GPR is the possibility of specifying the functional form of the covariance functions, which allows to introduce prior knowledge 
about the problem into the model. The training set $\mathbf{D}=\left\{\left(x_{1}, y_{1}\right),\left(x_{2}, y_{2}\right), \ldots,\left(x_{n}, y_{n}\right)\right\}$ is assumed to be drawn from the (noisy) process:

$$
y_{i}=f\left(x_{i}\right)+\varepsilon \quad \text { with } \varepsilon \sim N\left(0, \sigma^{2}\right)
$$

where $x_{i}$ is an input vector in $R^{d}$ and $y_{i}$ is a scalar output in $R^{1}$. Therefore we have a $R^{d} \rightarrow R^{1}$ mapping. For notational convenience, we aggregate the inputs and the outputs into matrix $\mathbf{X}=\left[x_{1}, x_{2}, \ldots, x_{n}\right]$ and $\mathbf{y}=\left[y_{1}, y_{2}, \ldots, y_{n}\right]$ respectively.

The joint distribution of the variables is the conditional Gaussian distribution:

$$
p(y / \mathbf{X}) \sim N\left(0, K(\mathbf{X}, \mathbf{X})+\sigma^{2} \mathbf{I}\right)
$$

where $I$ is the identity matrix, and the covariance matrix $\mathbf{K}(\mathbf{X}, \mathbf{X})$ is also called the kernel matrix with elements $K_{i j}\left(x_{i}, x_{j}\right)$. The kernel function $k\left(x, x^{\prime}\right)$ is a measure of the distance between input vectors.

For the kernel function, a common choice is the Gaussian, or squared exponential. In this study we make use of a radial basis kernel with a linear trend to account for the trend component present in most of the time series over the training period:

$$
K_{i j}=k\left(x_{i}, x_{j}\right)=v^{2} \exp \left(-\frac{\left(x_{i}-x_{j}\right)^{T}\left(x_{i}-x_{j}\right)}{2 \lambda^{2}}\right)+\gamma x_{i}{ }^{T} x_{j}+\kappa
$$

where $v^{2}$ controls the prior variance, and $\lambda$ is a parameter that controls the rate of decay of the covariance by determining how far away $x_{i}$ must be from $x_{j}$ for $f_{i}$ to be unrelated to $f_{j}$. Alternative sets of kernels are discussed in MacKay (2003). The hyperparameters $\{v, \lambda, \gamma, \kappa\}$ are estimated by maximum likelihood in:

$$
\log (p(y / x))=-\frac{1}{2} y^{T}\left[\mathbf{K}(\mathbf{X}, \mathbf{X})+\sigma^{2} \mathbf{I}\right]^{-1} y-\frac{1}{2} \log \left|\mathbf{K}(\mathbf{X}, \mathbf{X})+\sigma^{2} \mathbf{I}\right|-\frac{n}{2} \log 2 \pi
$$

Given the training samples $\left(x_{i}, y_{j}\right)$ and a set of test points $X *$, the objective of GPR is to find the predictive outputs $f^{*}$ with probabilistic confidence intervals. By making use of the Bayesian inference, the joint posterior distribution is:

$$
p\left(f, f^{*} / y\right)=\frac{p(y / f) p\left(f, f^{*}\right)}{p(y / X)}
$$

The joint prior distribution and the independent likelihood probability both follow a Gaussian distribution:

$$
p\left(f, f^{*}\right)=N\left(0,\left[\begin{array}{l}
K_{f, f} K_{f^{*}, f} \\
K_{f, f^{*}} K_{f^{*}, f^{*}}
\end{array}\right]\right)
$$




$$
p(y / f)=N\left(f, \sigma^{2} I\right)
$$

where $f$ and $f *$ are subscripts of the variables between which the covariance is

computed. The Gaussian predictive distribution $p\left(f^{*} / y\right)$ is characterized by mean $\mu$ and variance $\Sigma$.

Therefore the GPR model specification is given by equations:

$$
\begin{aligned}
& \mu=K\left(X^{*}, X\right)\left[\mathbf{K}(\mathbf{X}, \mathbf{X})+\sigma^{2} I\right]^{-1} y \\
& \Sigma=K\left(X^{*}, X^{*}\right)-K\left(X^{*}, X\right)\left[\mathbf{K}(\mathbf{X}, \mathbf{X})+\sigma^{2} I\right]^{-1} K\left(X, X^{*}\right)
\end{aligned}
$$

where $\mu$ is the predicted output, and the variance $\Sigma$ can be used to estimate confidence levels.

In this study we propose an extension of the model to multiple outputs based on an analogy to radial basis functions. We use a set of univariate predictors followed by a matrix product that takes into account the cross-dependencies of the outputs in order to improve the performance of the GPR. In this case we have a $R^{d} \rightarrow R^{M}$, where $M$ is the dimension of the output. This extension is applied by following a two-step training. First, we independently train each time series, generating supervised forecasts for each output. In the second step, by means of a regularized linear regression (Haykin, 2008), we generate forecasts for each output taking into account their correlations. This procedure is also applied to the NN models.

\subsection{Neural Network models}

\subsubsection{Radial Basis Function (RBF)}

Initially proposed by Broomhead and Lowe (1988), RBF networks are hybrid networks that combine both supervised and non-supervised learning. RBF NN are a special class of multi-layer feed-forward architecture with several layers of processing. First, an input layer, modelled as a feature vector of real numbers. Second, a hidden layer, which consists of a set of neurons, each of them computing a symmetric radial function centred each at a centroid $\mu_{j}$. Finally, an output layer that consists of a set of neurons, one for each given output. The output of the network can be expressed as a scalar function of the output vector of the hidden layer: 


$$
\begin{aligned}
& y_{t}=\beta_{0}+\sum_{j=1}^{q} \beta_{j} g_{j}\left(x_{t-i}\right) \\
& g_{j}\left(x_{t-i}\right)=\exp \left(-\frac{\sum_{j=1}^{p}\left(x_{t-i}-\mu_{j}\right)^{2}}{2 \sigma_{j}^{2}}\right) \\
& \left\{\begin{array}{l}
\left.x_{t-i} ; i=1, \ldots, p\right\} \\
\left.\beta_{j} ; \sigma_{\mathrm{j}} ; j=1, \ldots, q\right\}
\end{array}\right.
\end{aligned}
$$

where $y_{t}$ is the output vector of the network at time $t ; x_{t-i}$ is the input value at time $t-i$, where $i$ stands for the number of lags that are used to introduce the context of the actual observation; $g_{j}$ is the activation function, which usually has a Gaussian shape; $\beta_{j}$ are the weights connecting the output of the neuron $j$ at the hidden layer with the output neuron; $\mu_{j}$ is the centroid vector for neuron $j$; and the spread $\sigma_{j}$ is a scalar that measures the width over the input space of the Gaussian function. We denote $q$ as the number of neurons in the hidden layer, which ranges from 5 to 30 , increasing for longer forecasting horizons.

\subsubsection{Multi-layer Perceptron (MLP)}

MLP networks consist of multiple layers of computational units interconnected in a feed-forward way. MLP networks are supervised neural networks that use as a building block a simple perceptron model. The topology consists of layers of parallel perceptrons, with optimal connections between layers:

$$
\begin{aligned}
& y_{t}=\beta_{0}+\sum_{j=1}^{q} \beta_{j} g\left(\sum_{i=1}^{p} w_{i j} x_{t-i}+w_{0 j}\right) \\
& \left\{x_{t-i} ; i=1, \ldots, p\right\} \\
& \left\{w_{i j} ; i=1, \ldots, p ; j=1, \cdots, q\right\} \\
& \left\{\beta_{j} ; j=1, \ldots, q\right\}
\end{aligned}
$$

where $y_{t}$ is the output vector of the network at time $t ; x_{t-i}$ is the input value at time $t-i ; \beta_{j}$ are the weights connecting the output of the neuron $j$ at the hidden layer with the output neuron;. $w_{i j}$ stand for the weights of neuron $j$ connecting the input with the hidden layer; and $g$ is the non-linear function of the neurons in the hidden layer. The number of neurons in the hidden layer is denoted by $q$, and determines the network's capacity to approximate a given function. In order to solve the problem of overfitting, the number of neurons is estimated by cross-validation. 


\section{Forecasting comparison}

\subsection{Experimental design}

For an iterated multi-step-ahead forecasting comparison the partition between train and test sets is done sequentially: as the prediction advances, past forecasts are successively incorporated to the training database. As the size of the training set increases, for each predicted value in the test database, the first element of the validation database is transferred to the training database, and the last predicted value of the test database is incorporated to the validation database in a recursive way. Thus, the first ninety-six monthly observations are selected as the initial training set, the next $33 \%$ as the validation set, and the last $15 \%$ as the test set.

Once the topology of the neural networks is decided, the parameters of the networks are estimated by means of the Levenberg-Marquardt (LM) algorithm. In order to assure a correct performance of the RBF NNs, the number of centroids and the spread of each centroid have to be selected before the training phase. In this study the training is done by adding the centroids iteratively with the spread parameter fixed. Then a regularized linear regression is estimated to compute the connections between the hidden and the output layer. Finally, the performance of the networks is computed on the validation data set. This process is repeated until the performance on the validation database ceases to decrease. To avoid the possibility that the search for the optimum value of the parameters finishes in a local minimum, we use a multi-starting technique that initializes the NNs several times for different initial random values and returns the best result. All models are implemented with Python.

\subsection{Experimental results}

To assess the performance of the GPR model, we compare its forecasting accuracy to that of a RBF NN. We estimate the models and generate forecasts in a recursive way for different forecast horizons (1, 3, 6 and 12 months) during the out-of-sample period. In order to summarize the results of the forecasting comparison, we compute several forecast accuracy measures. First we obtain the Relative Mean Absolute Percentage Error (rMAPE) statistic for the GPR and the RBF NN with respect to a MLP NN model 
used as a benchmark (Table 1). Next, we run the Diebold-Mariano (DM) test using a Newey-West type estimator to analyse whether the reductions in MAPE between both models are statistically significant (Table 2). Finally, in Table 3 we compute the proportion of Periods with Lower Absolute Error (PLAE) statistic.

Table 1. Forecast accuracy. rMAPE - GPR and RBF NN vs. MLP NN

\begin{tabular}{|c|c|c|c|c|c|}
\hline & GPR & RBF NN & & GPR & RBF NN \\
\hline Andalusia & & & Valencia (Community) & & \\
\hline $\mathrm{h}=1$ & 0.823 & 0.921 & $\mathrm{~h}=1$ & 0.945 & 1.017 \\
\hline$h=3$ & 1.059 & 0.918 & $\mathrm{~h}=3$ & 0.948 & 0.924 \\
\hline$h=6$ & 0.971 & 0.795 & $h=6$ & 0.966 & 0.902 \\
\hline $\mathrm{h}=12$ & 1.197 & 0.769 & $h=12$ & 0.972 & 0.948 \\
\hline Aragon & & & Extremadura & & \\
\hline $\mathrm{h}=1$ & 0.820 & 0.935 & $\mathrm{~h}=1$ & 0.991 & 1.106 \\
\hline$h=3$ & 0.911 & 0.976 & $h=3$ & 1.228 & 1.307 \\
\hline$h=6$ & 1.041 & 0.928 & $h=6$ & 0.898 & 0.741 \\
\hline $\mathrm{h}=12$ & 0.866 & 0.850 & $\mathrm{~h}=12$ & 0.921 & 0.961 \\
\hline Asturias & & & Galicia & & \\
\hline $\mathrm{h}=1$ & 0.767 & 0.863 & $\mathrm{~h}=1$ & 0.845 & 0.931 \\
\hline $\mathrm{h}=3$ & 1.072 & 0.797 & $h=3$ & 1.068 & 0.760 \\
\hline$h=6$ & 0.871 & 0.895 & $h=6$ & 1.065 & 1.023 \\
\hline $\mathrm{h}=12$ & 0.859 & 0.758 & $\mathrm{~h}=12$ & 1.064 & 1.006 \\
\hline Balearic Islands & & & Madrid (Community) & & \\
\hline $\mathrm{h}=1$ & 0.746 & 0.755 & $\mathrm{~h}=1$ & 1.289 & 1.134 \\
\hline$h=3$ & 1.048 & 0.526 & $h=3$ & 1.049 & 1.092 \\
\hline$h=6$ & 1.112 & 1.507 & $\mathrm{~h}=6$ & 1.002 & 0.917 \\
\hline $\mathrm{h}=12$ & 2.359 & 1.671 & $\mathrm{~h}=12$ & 0.983 & 1.015 \\
\hline Canary Islands & & & Murcia (Region) & & \\
\hline $\mathrm{h}=1$ & 1.148 & 1.123 & $\mathrm{~h}=1$ & 1.061 & 1.121 \\
\hline$h=3$ & 1.002 & 1.003 & $h=3$ & 1.073 & 1.001 \\
\hline$h=6$ & 0.933 & 0.957 & $h=6$ & 0.920 & 0.845 \\
\hline $\mathrm{h}=12$ & 1.055 & 1.031 & $\mathrm{~h}=12$ & 0.919 & 0.836 \\
\hline Cantabria & & & Navarra & & \\
\hline $\mathrm{h}=1$ & 0.807 & 0.835 & $\mathrm{~h}=1$ & 0.798 & 0.928 \\
\hline$h=3$ & 0.910 & 0.715 & $h=3$ & 1.055 & 0.952 \\
\hline$h=6$ & 0.792 & 1.045 & $h=6$ & 1.080 & 1.082 \\
\hline$h=12$ & 0.712 & 0.586 & $\mathrm{~h}=12$ & 0.814 & 0.827 \\
\hline Castilla-Leon & & & Basque Country & & \\
\hline $\mathrm{h}=1$ & 0.761 & 0.966 & $\mathrm{~h}=1$ & 0.871 & 0.914 \\
\hline$h=3$ & 0.841 & 0.797 & $h=3$ & 0.909 & 0.914 \\
\hline$h=6$ & 0.935 & 0.933 & $\mathrm{~h}=6$ & 0.924 & 0.945 \\
\hline $\mathrm{h}=12$ & 0.913 & 0.818 & $\mathrm{~h}=12$ & 0.894 & 0.954 \\
\hline Castilla-La Mancha & & & La Rioja & & \\
\hline $\mathrm{h}=1$ & 0.592 & 0.862 & $\mathrm{~h}=1$ & 1.026 & 1.058 \\
\hline$h=3$ & 0.736 & 0.838 & $h=3$ & 0.769 & 0.613 \\
\hline$h=6$ & 0.916 & 0.911 & $h=6$ & 0.976 & 0.677 \\
\hline$h=12$ & 0.872 & 0.696 & $h=12$ & 1.079 & 0.852 \\
\hline \multicolumn{6}{|l|}{ Catalonia } \\
\hline $\mathrm{h}=1$ & 0.794 & 0.948 & & & \\
\hline$h=3$ & 1.063 & 0.996 & & & \\
\hline$h=6$ & 1.017 & 0.968 & & & \\
\hline $\mathrm{h}=12$ & 0.816 & 0.872 & & & \\
\hline
\end{tabular}


Table 2. DM test statistic - GPR vs. RBF NN

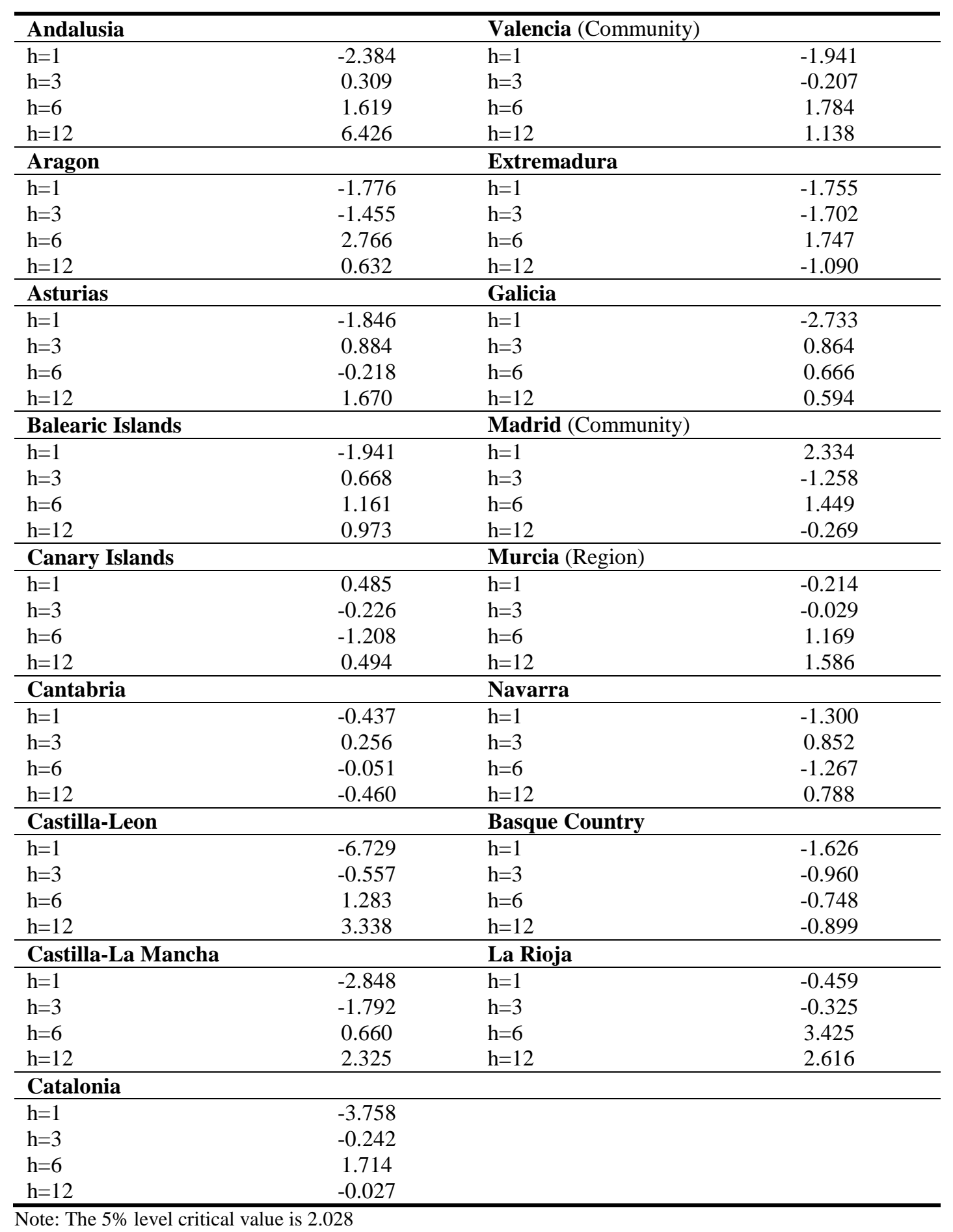

The results of the rMAPE for the GPR and the RBF NN models presented in Table 1 show that there are no major differences between both models when compared to a MLP NN. By regions, in the Balearic Islands, Madrid and the Canary Islands the MLP $\mathrm{NN}$ is rarely outperformed. In contrast, in Cantabria, Castilla-Leon, Castilla-La Mancha, and the Basque Country, both the GPR model and the RBF NN outperform the MLP for all forecast horizons. 
In order to test whether the differences between the two competing models are statistically significant, we calculate the DM test (Table 2). The null hypothesis of the test is that the difference between the two competing series is non-significant. A negative sign of the statistic implies that the RBF NN model has bigger forecasting errors.

The results of the DM test between the GPR and the RBF NN models presented in Table 2 indicate that only in $18 \%$ of the cases we find a significant difference between the absolute forecast errors of the GPR and the RBF NN model. In $58 \%$ of the cases, the RBF NN shows a significant improvement over the GPR. While in three regions (Cantabria, Catalonia and the Basque Country) the forecast errors of the RBF NN are bigger than the forecast errors of the GPR model, in the rest of the regions the results are mixed.

The improvement of the GPR model with respect to the RBF NN becomes more prominent for short-term forecast horizons (one and three-months ahead predictions). While for six and twelve-months ahead forecasts, the errors of the GPR are bigger than the ones of the RBF NN in 9 out of 17 regions (Andalusia, Aragon, the Balearic Islands, Castilla-Leon, Castilla-La Mancha, Valencia, Galicia, Murcia and La Rioja).

Finally, to attain a more comprehensive forecasting evaluation, we compute the PLAE statistic (Claveria et al., 2015a). The PLAE can be regarded as a variation of the Percent Better measure used in the M3-competition to compare the forecast accuracy of the models to a random walk (Makridakis and Hibon, 2000). The PLAE is a dimensionless measure based on the CJ statistic for testing market efficiency (Cowles and Jones, 1937). This accuracy measure allows us to compare the forecasting performance between two competing techniques against a benchmark model. In this study we use the MLP NN as a benchmark.

The PLAE statistic is a ratio that gives the proportion of periods in which the model under evaluation obtains lower absolute forecast errors than the benchmark model. Let us denote $y_{t}$ as actual value and $\hat{y}_{t}$ as forecast at period $t=1, \ldots, n$. Forecast errors can then be defined as $e_{t}=y_{t}-\hat{y}_{t}$. Given two competing models $A$ and $B$, where $A$ refers to the forecasting model under evaluation and $B$ stands for benchmark model, we can then obtain the proposed statistic as follows:

$$
P L A E=\frac{\sum_{t=1}^{n} \lambda_{t}}{n} \text { where } \lambda_{t}= \begin{cases}1 & \text { if }\left|e_{t, A}\right|<\left|e_{t, B}\right| \\ 0 & \text { otherwise }\end{cases}
$$


Table 3. Forecast accuracy. PLAE - GPR and RBF NN vs. MLP NN

\begin{tabular}{|c|c|c|c|c|c|}
\hline & GPR & RBF NN & & GPR & RBF NN \\
\hline Andalusia & & & Valencia (Community) & & \\
\hline $\mathrm{h}=1$ & 0.364 & 0.273 & $\mathrm{~h}=1$ & 0.182 & 0.910 \\
\hline$h=3$ & 0.273 & 0.545 & $h=3$ & 0.273 & 0.455 \\
\hline$h=6$ & 0.455 & 0.545 & $h=6$ & 0.364 & 0.455 \\
\hline$h=12$ & 0.818 & 0.818 & $h=12$ & 0.636 & 0.727 \\
\hline Aragon & & & Extremadura & & \\
\hline $\mathrm{h}=1$ & 0.273 & 0.273 & $\mathrm{~h}=1$ & 0.182 & 0.182 \\
\hline$h=3$ & 0.545 & 0.727 & $h=3$ & 0.273 & 0.727 \\
\hline $\mathrm{h}=6$ & 0.727 & 0.545 & $\mathrm{~h}=6$ & 0.727 & 0.818 \\
\hline $\mathrm{h}=12$ & 0.636 & 0.727 & $\mathrm{~h}=12$ & 0.909 & 0.818 \\
\hline Asturias & & & Galicia & & \\
\hline $\mathrm{h}=1$ & 0.182 & 0.182 & $\mathrm{~h}=1$ & 0.910 & 0.910 \\
\hline$h=3$ & 0.545 & 0.909 & $h=3$ & 0.636 & 0.818 \\
\hline $\mathrm{h}=6$ & 0.818 & 0.818 & $\mathrm{~h}=6$ & 0.818 & 0.909 \\
\hline $\mathrm{h}=12$ & 0.818 & 0.818 & $\mathrm{~h}=12$ & 0.909 & 0.909 \\
\hline Balearic Islands & & & Madrid (Community) & & \\
\hline $\mathrm{h}=1$ & 0.545 & 0.545 & $\mathrm{~h}=1$ & 0.000 & 0.182 \\
\hline$h=3$ & 0.818 & 0.909 & $h=3$ & 0.182 & 0.182 \\
\hline $\mathrm{h}=6$ & 0.909 & 1.000 & $\mathrm{~h}=6$ & 0.182 & 0.273 \\
\hline $\mathrm{h}=12$ & 1.000 & 1.000 & $\mathrm{~h}=12$ & 0.000 & 0.000 \\
\hline Canary Islands & & & Murcia (Region) & & \\
\hline $\mathrm{h}=1$ & 0.000 & 0.000 & $\mathrm{~h}=1$ & 0.910 & 0.182 \\
\hline$h=3$ & 0.000 & 0.000 & $h=3$ & 0.364 & 0.545 \\
\hline $\mathrm{h}=6$ & 0.000 & 0.000 & $\mathrm{~h}=6$ & 0.364 & 0.455 \\
\hline $\mathrm{h}=12$ & 0.000 & 0.000 & $\mathrm{~h}=12$ & 0.636 & 0.818 \\
\hline Cantabria & & & Navarra & & \\
\hline $\mathrm{h}=1$ & 0.364 & 0.364 & $\mathrm{~h}=1$ & 0.182 & 0.910 \\
\hline$h=3$ & 0.818 & 0.909 & $h=3$ & 0.545 & 0.818 \\
\hline $\mathrm{h}=6$ & 0.818 & 0.909 & $\mathrm{~h}=6$ & 0.636 & 0.727 \\
\hline$h=12$ & 1.000 & 0.909 & $\mathrm{~h}=12$ & 0.727 & 0.636 \\
\hline Castilla-Leon & & & Basque Country & & \\
\hline $\mathrm{h}=1$ & 0.545 & 0.910 & $\mathrm{~h}=1$ & 0.182 & 0.182 \\
\hline$h=3$ & 0.636 & 0.909 & $h=3$ & 0.273 & 0.455 \\
\hline $\mathrm{h}=6$ & 0.727 & 0.818 & $\mathrm{~h}=6$ & 0.545 & 0.455 \\
\hline $\mathrm{h}=12$ & 0.909 & 0.909 & $\mathrm{~h}=12$ & 0.910 & 0.273 \\
\hline Castilla-La Mancha & & & La Rioja & & \\
\hline $\mathrm{h}=1$ & 0.636 & 0.545 & $\mathrm{~h}=1$ & 0.182 & 0.273 \\
\hline$h=3$ & 0.727 & 0.909 & $h=3$ & 0.727 & 0.909 \\
\hline $\mathrm{h}=6$ & 0.818 & 0.818 & $h=6$ & 0.727 & 0.727 \\
\hline$h=12$ & 0.818 & 0.818 & $\mathrm{~h}=12$ & 0.818 & 0.909 \\
\hline \multicolumn{6}{|l|}{ Catalonia } \\
\hline $\mathrm{h}=1$ & 0.273 & 0.910 & & & \\
\hline$h=3$ & 0.36 .4 & 0.545 & & & \\
\hline $\mathrm{h}=6$ & 0.818 & 0.818 & & & \\
\hline $\mathrm{h}=12$ & 0.727 & 0.727 & & & \\
\hline
\end{tabular}

Note: The PLAE ratio measures the proportion of out-of-sample periods with lower absolute errors than the benchmark model (MLP NN model). Values below 0.5 indicate that the benchmark model displays a higher number of lower absolute forecast errors than the model under evaluation for the out-of-sample period. 
Table 3 shows the results of the PLAE statistic for the GPR and the RBF NN compared to the MLP NN. We do not find relevant differences between the GPR and the RBF NN when compared to the MLP NN. Both the GPR and the RBF NN display higher PLAE values than the MLP NN for all forecast horizons except for one-month ahead predictions, where the MLP NN shows a higher proportion of out-of-sample periods with lower absolute errors in all regions except two (the Balearic Islands and Castilla-La Mancha). Special mention should be made to the Canary Islands and the Community of Madrid, where neither model outperforms the MLP NN regardless of the forecast horizon. These results are in line with those obtained in Table 1.

In order to evaluate the effect of the memory on the forecasting results, we repeat the experiment considering different topologies regarding the number of lags used for concatenation. In Table 4 we present the results of the rMAPE and the DM test for the GPR model with a memory of one period with respect to the GPR with $i=3$. We find that when additional lags are incorporated in the feature vector, the rMAPE results show that the forecasting performance of the GPR models improves in almost $70 \%$ of the cases.

Finally, in Table 4 we also present the results of the DM test between the GPR with a one-period memory and the GPR with a three-period memory. We find that in $54 \%$ of the cases there is a significant difference between the absolute forecasting errors of the GPR for $i=1$ and the GPR for $i=3$. In $90 \%$ of the cases, incorporating additional lags in the model results in a significant improvement. Madrid and the Canary Islands are the only regions where there is no significant reduction in forecasting errors when increasing the memory of the model. The fact that both regions are the ones with the lowest temporal concentration of tourism demand suggests that increasing the memory of the models is particularly indicated when the series present a marked seasonal component. This evidence is in line with the results obtained by Claveria et al. (2016a), who found that GPR models could not outperform naïve forecasts in the absence of seasonality regardless of the forecast horizon.

Overall, the empirical experiment shows that the forecasting performance of the different techniques improves for longer forecast horizons. For the Balearic Islands, Palmer et al. (2006) found that NNs were especially suitable for long-term forecasting, which is in line with previous research by Burger et al. (2001), Pattie and Snyder (1996) and Teräsvirta et al. (2005). However, we find that the RBF NN generates better predictions than the GPR models when compared to a MLP NN, especially for longer- 
term forecast horizons. This output suggests that RBF networks are better able to capture the seasonal pattern of the series than interpolation methods such as the GPR model. Cang (2014), Claveria et al. (2015a,b) and Çuhadar et al. (2014) also obtained better results with RBF networks than with other $\mathrm{NN}$ architectures for seasonal forecasting.

Table 4. Forecast accuracy. rMAPE and DM test statistic - GPR( $i=1)$ vs. $\operatorname{GPR}(i=3)$

\begin{tabular}{|c|c|c|c|c|c|}
\hline & rMAPE & DM & & rMAPE & $\mathrm{DM}$ \\
\hline Andalusia & & & Valencia (Community) & & \\
\hline $\mathrm{h}=1$ & 1.264 & -3.828 & $\mathrm{~h}=1$ & 1.162 & -1.341 \\
\hline$h=3$ & 1.685 & -5.386 & $h=3$ & 1.231 & -2.429 \\
\hline$h=6$ & 1.151 & -4.619 & $h=6$ & 1.123 & -3.153 \\
\hline $\mathrm{h}=12$ & 1.498 & -2.113 & $\mathrm{~h}=12$ & 1.084 & -2.744 \\
\hline Aragon & & & Extremadura & & \\
\hline $\mathrm{h}=1$ & 0.914 & -0.376 & $\mathrm{~h}=1$ & 1.048 & -0.685 \\
\hline $\mathrm{h}=3$ & 1.200 & -2.204 & $h=3$ & 1.374 & -1.863 \\
\hline$h=6$ & 1.022 & -2.294 & $h=6$ & 0.930 & -2.259 \\
\hline $\mathrm{h}=12$ & 1.089 & 2.192 & $\mathrm{~h}=12$ & 0.827 & -1.933 \\
\hline Asturias & & & Galicia & & \\
\hline $\mathrm{h}=1$ & 0.805 & -1.301 & $\mathrm{~h}=1$ & 0.866 & -1.536 \\
\hline$h=3$ & 1.569 & -2.823 & $h=3$ & 1.188 & -3.409 \\
\hline $\mathrm{h}=6$ & 1.149 & -2.517 & $\mathrm{~h}=6$ & 0.988 & -2.314 \\
\hline $\mathrm{h}=12$ & 1.108 & 0.660 & $\mathrm{~h}=12$ & 0.759 & -0.400 \\
\hline Balearic Islands & & & Madrid (Community) & & \\
\hline $\mathrm{h}=1$ & 0.770 & -1.102 & $\mathrm{~h}=1$ & 1.206 & 0.361 \\
\hline$h=3$ & 1.378 & -3.404 & $h=3$ & 1.123 & 0.325 \\
\hline$h=6$ & 0.529 & -3.553 & $h=6$ & 1.066 & 0.950 \\
\hline $\mathrm{h}=12$ & 0.964 & -0.239 & $\mathrm{~h}=12$ & 1.023 & 0.962 \\
\hline Canary Islands & & & Murcia (Region) & & \\
\hline $\mathrm{h}=1$ & 0.960 & 2.768 & $\mathrm{~h}=1$ & 1.208 & -0.007 \\
\hline$h=3$ & 0.947 & 0.891 & $\mathrm{~h}=3$ & 1.641 & -3.069 \\
\hline$h=6$ & 1.042 & 0.256 & $\mathrm{~h}=6$ & 1.263 & -4.365 \\
\hline $\mathrm{h}=12$ & 1.092 & -0.898 & $\mathrm{~h}=12$ & 1.088 & -3.397 \\
\hline Cantabria & & & Navarra & & \\
\hline $\mathrm{h}=1$ & 0.944 & -2.499 & $\mathrm{~h}=1$ & 0.767 & -1.395 \\
\hline$h=3$ & 1.396 & -3.326 & $h=3$ & 1.356 & -3.052 \\
\hline$h=6$ & 1.062 & -3.798 & $h=6$ & 1.021 & -2.534 \\
\hline$h=12$ & 0.940 & 0.058 & $\mathrm{~h}=12$ & 0.843 & 2.110 \\
\hline Castilla-Leon & & & Basque Country & & \\
\hline $\mathrm{h}=1$ & 0.855 & -1.948 & $\mathrm{~h}=1$ & 1.049 & -2.142 \\
\hline$h=3$ & 1.147 & -4.885 & $h=3$ & 1.160 & -1.760 \\
\hline$h=6$ & 0.871 & -3.150 & $h=6$ & 1.066 & -1.416 \\
\hline$h=12$ & 0.875 & 1.040 & $\mathrm{~h}=12$ & 1.071 & 1.008 \\
\hline Castilla-La Mancha & & & La Rioja & & \\
\hline $\mathrm{h}=1$ & 1.006 & -2.987 & $\mathrm{~h}=1$ & 0.932 & -0.533 \\
\hline$h=3$ & 1.250 & -4.548 & $h=3$ & 1.246 & -3.585 \\
\hline$h=6$ & 1.165 & -3.781 & $\mathrm{~h}=6$ & 1.001 & -3.221 \\
\hline$h=12$ & 0.859 & -2.239 & $\mathrm{~h}=12$ & 1.276 & -0.046 \\
\hline \multicolumn{6}{|l|}{ Catalonia } \\
\hline $\mathrm{h}=1$ & 0.887 & -1.635 & & & \\
\hline$h=3$ & 1.372 & -2.107 & & & \\
\hline$h=6$ & 1.055 & -1.683 & & & \\
\hline $\mathrm{h}=12$ & 1.068 & 2.405 & & & \\
\hline
\end{tabular}

Note: The $5 \%$ level critical value is 2.028 
When comparing the forecasting accuracy of a sparse GPR model than with ARMA and SVR models for tourist arrivals to Hong Kong, Wu et al. (2012) obtained better forecasting results with the GPR model. In our case, the GPR model only outperformed the RBF NN for short-term forecast horizons. Nevertheless, there are several differences between both approaches. On the one hand, the models used in the forecasting comparison differ between both studies. In this experiment we compared a GPR model to a RBF NN architecture, using a MLP NN model as a benchmark. Moreover, we did not apply any sparse approximation to reduce the computational complexity of the GPR model due to the size of the sample. On the other hand, we applied a MIMO setting instead of a single-input single-output approach. In this sense, Ben Taieb et al. (2010) and Claveria et al. (2015a) also found evidence that MIMO strategies for ML techniques were particularly suitable for long-term forecasting.

\section{Concluding remarks}

In this study we present a multiple-input multiple-output (MIMO) framework for Gaussian process regression (GPR). In order to examine the predictive performance of the proposed method we compare its out-of-sample forecast accuracy to that of two neural network architectures (RBF NN and MLP NN) in a multiple-step-ahead forecasting comparison. The MIMO forecasting strategy allows modelling the interdependencies between the inputs in order to generate a vector of future values. By using the cross-correlations between tourist arrivals to all seventeen regions of Spain we forecast tourist demand for all markets simultaneously.

The forecasting results show that the proposed extension of the GPR only outperforms the $\mathrm{NN}$ models for short-term forecasts. We find that the predictive performance of all techniques improves for for the longest forecast horizons, which suggests that machine learning techniques are specially suitable for mid and long-term forecasting.

To evaluate the effect of an increase in the dimensionality of the input on forecast accuracy, we repeat the experiment by increasing the temporal context. As we increase the number of lags used for concatenation, we find that the forecasting performance of MIMO GPR models improves. This finding shows that the increase in the weight matrix is compensated by a more complex specification, and highlights the convenience of 
designing a model selection criteria to estimate the optimal number of lags when forecasting with machine learning methods.

The assessment of alternative kernel functions that can support a broader class of covariance functions on the forecasting accuracy of GPR models is a question to be addressed in further research. Another question to be considered in future research is the effect of different sparse approximations for parameter estimation on forecast accuracy.

\section{References}

Adya M, Collopy F (1998) How effective are neural networks at forecasting and prediction? A review and evaluation. Journal of Forecasting 17: 481-495.

Aguiló E (2010) Una panorámica de la economía del turismo en España. Cuadernos de Economía 33: 5-42.

Aguiló E, Riera A, Rosselló J (2005) The short-term price effect of a tourist tax through a dynamic demand model: The Case of the Balearic Islands. Tourism Management 26: 359365.

Ahmed NK, Atiya AF, El Gayar N, El-Shishiny H (2010) An empirical comparison of machine learning models for time series forecasting. Econometric Reviews 29:594-621.

Akin M (2015) A novel approach to model selection in tourism demand modeling. Tourism Management 48: 64-72.

Álvarez-Díaz M, Rosselló-Nadal J (2010) Forecasting British tourist arrivals in the Balearic Islands using meteorological variables. Tourism Economics 16: 153-168.

Andrades-Caldito L, Sánchez-Rivero M, Pulido-Fernández JI (2013) Differentiating competitiveness through tourism image assessment. An application to Andalusia (Spain). Journal of Travel Research 52: 68-81.

Andrawis RR, Atiya AF, El-Shishiny H (2011) Forecast combinations of computational intelligence and linear models for the NN5 time series forecasting competition. International Journal of Forecasting 27: 672-688.

Aminian F, Dante E, Aminian M, Waltz D (2006) Forecasting economic data with neural networks. Computational Economics 28: 71-88.

Athanasopoulos G, Hyndman RJ, Song H, Wu DC (2011) The tourism forecasting competition. International Journal of Forecasting 27: 822-844.

Balaguer J, Cantavella-Jordá M (2002) Tourism as a long-run economic growth tactor: The Spanish case. Applied Economics 34: 877-884.

Banerjee S, Gelfand AE, Finley AO, Sang H (2008) Gaussian predictive process models for large spatial data sets. Journal of the Royal Statistical Society: Series B (Statistical Methodology), 70: 825-848.

Bardolet E, Sheldon PJ (2008) Tourism in archipelagos. Hawai'i and the Balearics. Annals of Tourism Research 35: 900-923.

Ben Taieb S, Sorjamaa A, Bontempi G (2010) Multiple-output Modeling for Multi-step-ahead Time Series Forecasting. Neurocomputing 73: 1950-1957.

Biagi B, Pulina M (2009) Bivariate VAR models to test Granger causality between tourist demand and supply: Implications for regional sustainable growth. Papers in Regional Science 88: 231-244.

Brahim-Belhouari S, Bermak A (2004) Gaussian process for nonstationary time series prediction. Computational Statistics \& Data Analysis 47: 705-712.

Broomhead DS., Lowe D (1988) Multi-variable functional interpolation and adaptive networks. Complex Syst. 2: 321-355. 
Burger C, Dohnal M, Kathrada M, Law R (2001) A practitioners guide to time-series methods for tourism demand forecasting: A case study of Durban, South Africa. Tourism Management 22: 403-409.

Cang S (2014) A Comparative analysis of three types of tourism demand forecasting models: Individual, linear combination and non-linear combination. International Journal of Tourism Research 16: 596-607.

Capó J, Riera A, Rosselló J (2007). Tourism and long-term growth. A Spanish perspective. Annals of Tourism Research 34: 709-726.

Chapados, N., Bengio, Y., 2007. Augmented functional time series representation and forecasting with Gaussian processes. In B. Schölkopf, J. C. Platt, \& T. Hoffman (Eds.), Advances in Neural Information Processing Systems, 19 (pp. 457-464). The MIT Press, Cambridge, MA.

Chen AS, Leung MT (2005) Performance evaluation of neural network architectures: The case of predicting foreign exchange correlations. Journal of Forecasting 24: 403-420.

Chen KY, Wang CH (2007) Support vector regression with genetic algorithms in forecasting tourism demand. Tourism Management 28: 215-226.

Cho V (2003) A comparison of three different approaches to tourist arrival forecasting. Tourism Management 24: 323-330.

Chou MC (2013) Does tourism development promote economic growth in transition countries? A panel data analysis. Economic Modelling 33: 226-232.

Claveria O, Torra S (2014) Forecasting tourism demand to Catalonia: Neural networks vs. time series models. Economic Modelling 36: 220-228.

Claveria O, Monte E, Torra S (2015a) A new forecasting approach for the hospitality industry. International Journal of Contemporary Hospitality Management 27: 1520-1538.

Claveria O, Monte E, Torra S (2015b) Tourism demand forecasting with neural network models: Different ways of treating information. International Journal of Tourism Research 17: 492-500.

Claveria O, Monte E, Torra S (2016a) Modelling cross-dependencies between Spain's regional tourism markets with an extension of the Gaussian process regression model. SERIES 7: 341-357.

Claveria O, Monte E, Torra S (2016b) Modelling tourism demand to Spain with machine learning techniques. The impact of forecast horizon on model selection. Revista de Economía Aplicada 24(72): 109-132.

Claveria O, Monte E, Torra S (2017) Data pre-processing for neural network-based forecasting: Does it really matter? Technical and Economic Development of Economy. Forthcoming.

Cowles A, Jones H (1937) Some a posteriori probabilities in stock market action. Econometrica 5: 280-294.

Çuhadar M, Cogurcu I, Kukrer C (2014) Modelling and forecasting cruise tourism demand to İzmir by different artificial neural network architectures. International Journal of Business and Social Research 4: 12-28.

De la Mata R, Llano-Verduras E (2012) Spatial pattern and domestic tourism: An econometric analysis using inter-regional monetary flows by type of journey. Papers in Regional Science 91: 437-470.

Durbarry R (2004) Tourism and economic growth: the case of Mauritius. Tourism Economics 10: 389-401.

Duro JA, Farré FX (2015) Estacionalidad turística en las provincias españolas: Medición y análisis. Cuadernos de Turismo 36: 157-174.

Garín-Muñoz T, Montero-Martín LF (2007) Tourism in the Balearic Islands: A dynamic model for international demand using panel data. Tourism Management 28: 1224-1235.

Gil-Alana LA (2010) International arrivals in the Canary Islands: Persistence, long memory, seasonality and other implicit dynamics. Tourism Economics 16: 287-302.

Gil-Alana LA, Cunado J, De Gracia FP (2008) Tourism in the Canary Islands: Forecasting using several seasonal time series models. Journal of Forecasting 27: 621-636.

Girard A, Rasmussen C, Quiñonero-Candela J, Murray-Smith R (2003) Multiple-step ahead prediction for non linear dynamic systems - a Gaussian process treatment with propagation 
of the uncertainty. In S. Becker, S. Thrun, \& K. Obermayer (Eds.), Advances in Neural Information Processing Systems 15. The MIT Press, Cambridge, MA.

Guizzardi A, Stacchini A (2015) Real-time forecasting regional tourism with business sentiment surveys. Tourism Management 47: 213-223.

Haykin S (2008) Neural Networks and Learning Machines. Prentice Hall, New Jersey.

Hong W, Dong Y, Chen L, Wei S (2011) SVR with hybrid chaotic genetic algorithms for tourism demand forecasting. Applied Soft Computing 11: 1881-1890.

Kock AB, Teräsvirta T (2014) Forecasting performances of three automated modelling techniques during the economic crisis 2007-2009. International Journal of Forecasting 30: 616-631.

Kon S, Turner LL (2005) Neural network forecasting of tourism demand. Tourism Economics 11: 301-328.

Krige DG (1951) A statistical approach to some basic mine valuation problems on the Witwatersrand. Journal of the Chemistry, Metallurgical and Mining Society of South Africa 52: 119-139.

Law R (2000) Back-propagation learning in improving the accuracy of neural network-based tourism demand forecasting. Tourism Management 21: 331-340.

Ledesma-Rodríguez FJ, Navarro-Ibanez M, Pérez-Rodríguez JV (2001) Panel data and tourism: A case study of Tenerife. Tourism Economics 7: 75-88.

Lehmann R, Wohlrabe K. 2015. Forecasting GDP at the regional level with many predictors. German Economic Review 16: 226-254.

Lin C, Chen H, Lee T (2011) Forecasting tourism demand using time series, artificial neural networks and multivariate adaptive regression splines: Evidence from Taiwan. International Journal of Business Administration 2: 14-24.

MacKay DJC (2003) Information Theory, Inference, and Learning Algorithms. Cambridge University Press: Cambridge.

Makridakis S, Hibon M (2000) The M3-competition: results, conclusions and implications. International Journal of Forecasting 16: 451-476.

Matheron G (1973) The intrinsic random functions and their applications. Advances in Applied Probability 5: 439-468.

Medeiros MC, Teräsvirta T, Rech G (2006) Building neural network models for time series: A statistical approach. Journal of Forecasting 25: 49-75.

Medeiros MC, McAleer M, Slottje D, Ramos V, Rey-Maquieira J (2008) An alternative approach to estimating demand: Neural network regression with conditional volatility for high frequency air passenger arrivals. Journal of Econometrics 147: 372-383.

Molinet T, Molinet JA, Betancourt ME, Palmer A, Montaño JJ (2015) Models of artificial neural networks applied to demand forecasting in nonconsolidated tourist destinations. Methodology: European Journal of Research Methods for the Behavioral and Social Sciences 11: 35-44.

Nelson M, Hill T, Remus W, O’Connor M (1999) Time series forecasting using neural networks: Should the data be deseasonalized first?. Journal of Forecasting 18: 359-367.

Ortega L, Khashanah K (2014) A neuro-wavelet model for the short-term forecasting of highfrequency time series of stock returns. Journal of Forecasting 33: 134-146.

Paci R, Marrocu E (2014) Tourism and regional growth in Europe. Papers in Regional Science 93(S1): S25-S50.

Padhi SS, Aggarwal V (2011) Competitive revenue management for fixing quota and price of hotel commodities under uncertainty. International Journal of Hospitality Management 30: $725-734$.

Palmer A, Montaño JJ, Sesé A (2006) Designing an artificial neural network for forecasting tourism time-series. Tourism Management 27: 781-790.

Pattie DC, Snyder J (1996) Using a neural network to forecast visitor behavior. Annals of Tourism Research 23: 151-164.

Peng B, Song H, Crouch GI (2014) A Meta-analysis of international tourism demand forecasting and implications for practice. Tourism Management 45: 181-193.

Pérez-Rodríguez JV, Ledesma-Rodríguez F, Santana-Gallego M (2015) Testing dependence between GDP and tourism's growth rates. Tourism Management 48: 268-282. 
Priego FJ, Rosselló J, Santana-Gallego M (2015) The impact of climate change on domestic tourism: a gravity model for Spain. Regional Environmental Change 15: 291-300.

Quiñonero-Candela J, Rasmussen CE (2005) A unifying view of sparse approximate Gaussian process regression. Journal of Machine Learning Research 6: 1939-1959.

Rasmussen CE (1996) The infinite Gaussian mixture model. Advances in Neural Information Processing Systems 8: 514-520.

Rasmussen CE, Williams CKI (2006) Gaussian Processes for Machine Learning. The MIT Press: Cambridge, MA.

Rickman DS, Miller SR, McKenzie R (2009) Spatial and sectoral linkages in regional models: A Bayesian vector autoregression forecast evaluation. Papers in Regional Science 88: 29 41.

Rosselló J, Riera A, Sansó A (2004) The economic determinants of seasonal patterns. Annals of Tourism Research 31: 697-711.

Rosselló-Nadal J (2001) Forecasting turning points in international visitor arrivals in the Balearic Islands. Tourism Economics 7: 365-380.

Saenz-de-Miera O, Rosselló J (2014) Modeling tourism impacts on air pollution: the case study of $P M_{10}$ in Mallorca. Tourism Management 40: 273-281.

Sánchez M, Cárdenas PJ, Pulido JI (2015) Does tourism growth influence economic development? Journal of Travel Research 54: 206-221.

Santana-Jiménez Y, Hernández JM (2011) Estimating the effect of overcrowding on tourist attraction: The case of Canary Islands. Tourism Management 32: 415-425.

Sarrión-Gavilán MD, Benítez-Márquez MD, Mora-Rangel EO (2015) Spatial distribution of tourism supply in Andalusia. Tourism Management 15: 29-45.

Schubert SF, Brida JG, Risso WA (2011). The impacts of international tourism demand on economic growth of small economies dependent on tourism. Tourism Management 32: 377-385.

Smola AJ, Bartlett PL (2001) Sparse greedy Gaussian process regression. In T. K. Leen, T. G. Dietterich, and V. Tresp (Eds.) Advances in Neural Information Processing Systems 13 (619-625). The MIT Press: Cambridge, MA.

Song H, Li G (2008) Tourism demand modelling and forecasting - A review of recent research. Tourism Management 29: 203-220.

Stasinakis C, Sermpinis G, Theofilaos K, Karathanasopoulos A (2014) Forecasting US unemployment with radial basis neural networks, Kalman filters and support vector regressions. Computational Economics. Forthcoming

Teixeira JP, Fernandes PO (2012) Tourism time series forecast - Different ANN architectures with time index input. Procedia Technology 5: 445-454.

Tsaur S, Chiu Y, Huang C (2002) Determinants of guest loyalty to international tourist hotels: A neural network approach. Tourism Management 23: 397-405.

Uysal M, El Roubi MS (1999) Artificial neural networks versus multiple regression in tourism demand analysis. Journal of Travel Research 38: 111-118.

Vera JF, Ivars JA (2009) Spread of low-cost carriers: Tourism and regional policy effects in Spain. Regional Studies 43: 559-570.

Von Spreckelsen C, Von Mettenheim HJ, Breitner MH (2014) Real-time pricing and hedging of options on currency futures with artificial neural networks. Journal of Forecasting 33: 419-432.

Wu Q, Law R, Xu X (2012) A spare Gaussian process regression model for tourism demand forecasting in Hong Kong. Expert Systems with Applications 39: 4769-4774.

Xu X, Law R, Chen W, Tang L (2016) Forecasting tourism demand by extracting fuzzy Takagie-Sugeno rules from trained SVMs. CAAI Transactions on Intelligence Technology 1: $30-42$.

Yang D, Chaojun G, Dong Z, Jirutitijaroen P, Chen N, Walsh WM (2013) Solar irradiance forecasting using spatial-temporal covariance structures and time-forward kriging. Renewable Energy 60: 235-245.

Zhang G, Putuwo BE, Hu MY (1998) Forecasting with artificial neural networks: the state of the art. International Journal of Forecasting 14: 35-62. 


\section{$\underset{\oplus}{\mathbb{Q}}|\mathrm{R}| \mathrm{E}|\mathrm{A}|$}

Institut de Recerca en Economia Aplicada Regional i Públic

Research Institute of Applied Economics

WEBSITE: www.ub-irea.com • CONTACT: irea@ub.edu

\section{AQR}

Grup de Recerca Anàlisi Quantitativa Regional

Regional Quantitative Analysis Research Group

WEBSITE: www.ub.edu/aqr/•CONTACT: aqr@ub.edu

\section{Universitat de Barcelona}

Av. Diagonal, 690 • 08034 Barcelona 